\title{
A Fine Pitch Bump Bonding Process Compatible With the Manufacture of the Pixel-HPD's for the LHCb RICH Detector
}

M. Campbell, G. Aglieri Rinella, G. Arnau Izquierdo, T. Gys, N. Kanaya, C. Newby, D. Piedigrossi, P. Riedler, J. Salmi, J. Salonen, S. Savolainen-Pulli, L. Somerville, I. Suni, S. Vahanen, and K. Wyllie

\begin{abstract}
A new approach to photo detection using pixel $\mathbf{H y}$ brid Photon Detectors (pixel-HPD's) has been adopted for the LHCb-RICH detector. These devices use a hybrid pixel detector inside an evacuated photo tube providing high-precision, low noise detection of Cherenkov radiation. The approach takes advantage of modern CMOS technology offering many advantages over more traditional techniques. These advantages include extremely high sensitivity, low noise and fast readout. A major technological challenge was the encapsulation of a hybrid pixel detector inside a photo detector tube. The fabrication of the HPD tube involves packaging of the pixel detector assembly onto a ceramic carrier to form the photo-anode and the subsequent bake out of the pixel anode under vacuum. Both processes involve high temperatures. A fine pitch solder bump-bonding technique, which is compatible with the manufacture of hybrid photo detectors, has been developed. The technology and the tests used for qualifying the new process for pixel-HPD production are described. More than 40 pixel detector assemblies have been produced with almost all showing $<1 \%$ of missing pixels. A number of assemblies were baked out using temperature cycles identical to those used for pixel-HPD manufacture. None of the assemblies demonstrated any degradation. SEM photos clearly indicate the reliability of the process. 10 pixel-HPD tubes have been produced using this new bump-bonding process and those behave according to expectations. The pixel-HPD is the first of a new generation of photo detector tubes suitable for RICH and other visible photon sensing applications.
\end{abstract}

Index Terms-Photodetectors, radiation detectors.

\section{INTRODUCTION}

$\mathbf{T}$ HE Ring Imaging Cherenkov detector (RICH) of the LHCb experiment [1] is a critical component in particle identification. The requirements of the detector are high sensitivity to single visible photons, $>70 \%$ packing density, $2.5 \mathrm{~mm} \times 2.5 \mathrm{~mm}$ granularity, the unambiguous association of hits with a single $25 \mathrm{~ns}$ bunch crossing, few false hits and the ability to handle a maximum trigger rate of $\sim 1 \mathrm{MHz}$. Moreover, the detectors have to cope with a total ionizing dose of $\sim 25 \mathrm{kRad}$ and operate in a magnetic field of up to 30 Gauss.

Manuscript received October 20, 2004; revised May 30, 2006.

M. Campbell, G. Aglieri-Rinella, G. Arnau-Izquierdo, T. Gys, N. Kanaya, D. Piedigrossi, P. Riedler, and K. Wyllie are with CERN, 1211 Geneva 23, Switzerland (e-mail: Michael.Campbell@cern.ch).

C. Newby and L. Somerville are with the Sub-department of Particle Physics, University of Oxford, Oxford OX1 3RH, U.K.

J. Salmi, J. Salonen, S. Savolainen-Pulli, I. Suni, and S. Vahanen are with VTT Information Technology, Microelectronic, Espoo FIN-02044 VTT, Finland.

Digital Object Identifier 10.1109/TNS.2006.880537

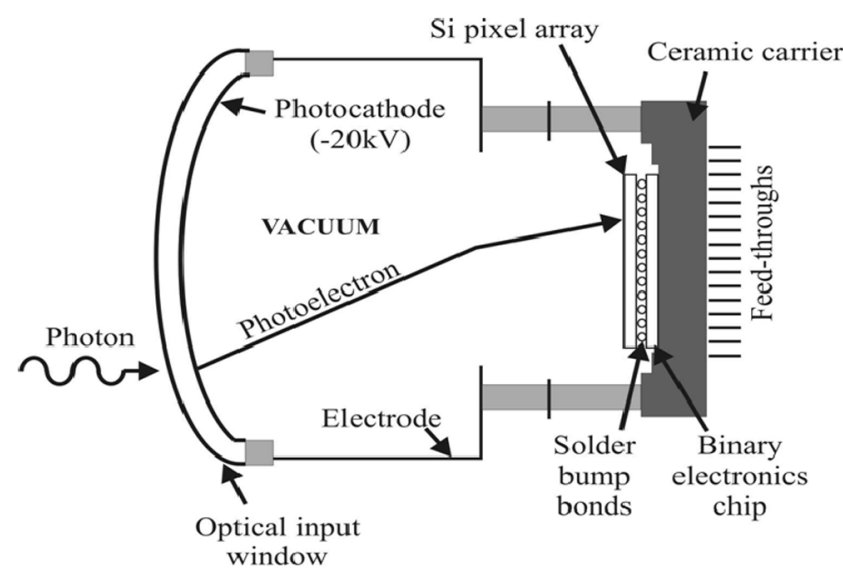

Fig. 1. The principle of operation of the pixel-HPD tube. A photon impinging on the photo-cathode generates a photoelectron which is accelerated and focused on the photo-anode in such a way that there is a $\times 5$ demagnification from the cathode entrance window to the photo-anode.

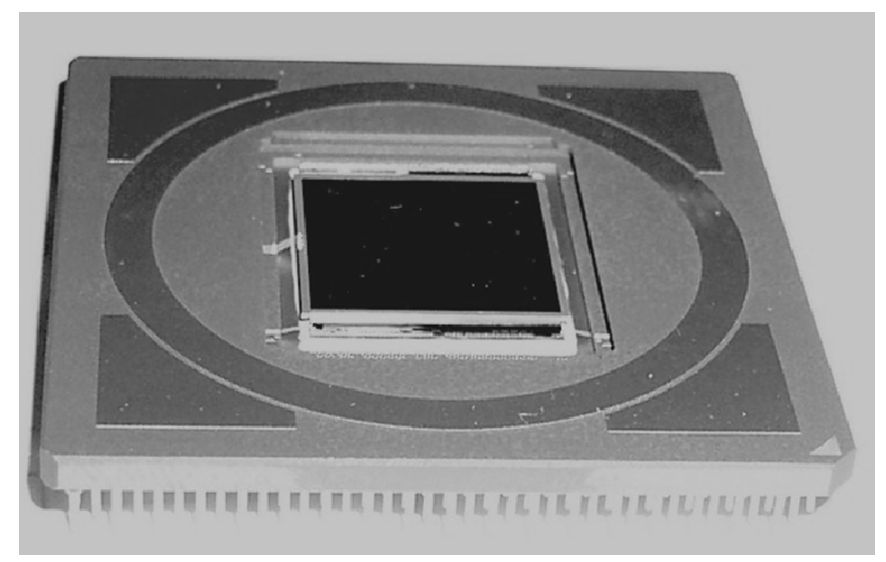

Fig. 2. A photo of a pixel-HPD anode. A tested silicon pixel assembly has been brazed onto a custom designed ceramic carrier prior to encapsulation in the HPD tube.

The pixel Hybrid Photon Detector (pixel-HPD) [2], [3] is the solution chosen for the LHCb RICH detector. Visible photons create photoelectrons when they impinge on the photo-cathode. Cross focusing electron optics allows for a $\mathrm{x} 5$ demagnification of the input picture onto $0.5 \mathrm{~mm}$ square elements on the anode, see Fig. 1. Single photoelectrons, which are accelerated by a $20 \mathrm{kV}$ bias voltage, impinge on the back of a pixellated high resistivity silicon sensor creating $\sim 5000$ electron-hole pairs in the substrate. Those charges drift towards collecting electrodes under the influence of an electric field which is applied between the entry window of the sensor and the inputs of a pixel readout 
ASIC. The ASIC [4], which is manufactured in a commercial $0.25 \mu \mathrm{m}$ process, provides low-noise pre-amplification and discrimination for single photo-electrons (the pre-amplifier input referred noise is $\sim 100 \mathrm{e}^{-} \mathrm{rms}$ and the discriminator threshold is $\sim 1500 \mathrm{e}^{-}$), single bunch crossing time tagging and a 16-event derandomizing buffer for triggered events. In fact, the $0.5 \mathrm{~mm}$ square elements are in reality made of 8 equally sized rectangular pixels whose discriminator outputs are OR-ed together [4]. Readout of triggered events is performed simultaneously with the detection of incoming incident photons.

A number of technological challenges had to be overcome in order to produce this new photon detector. In particular, the hybrid pixel assembly has to be encapsulated in the pixel-HPD tube, a process which involves two high temperature heat cycles. Standard tin-lead eutectic solder bump-bonding proved to be unsuitable for this application. A new high melting point bump bonding process has been developed and the technology and the results are presented in the paper.

The paper begins by describing the steps required to fabricate the pixel-HPD tube outlining the requirements on the solder bump-bonding process. Modifications to the standard eutectic bump-bonding process will then be outlined. Bake out tests were performed on eutectic assemblies and on assemblies made using the high-Pb bumps and the results of those tests will be described and summarized. Finally, a number of pixel-HPD tubes have been produced and first results are shown.

\section{OUtLINE OF THE FABRICATION PROCEEDURE FOR THE PIXEL-HPD}

The fabrication of pixel-HPD's is carried out mostly in European industry and coordinated by a group at CERN. The LHCBPIX1 ASIC [4] is designed in a commercial $0.25 \mu \mathrm{m}$ process and therefore chips are manufactured on $200 \mathrm{~mm}$ CMOS wafers. The CMOS wafers are probe tested at CERN to select Known Good Die for bump bonding to sensors. The ASIC is designed such that every pixel in the matrix can be tested individually and its threshold and input referred noise measured. During these tests around 55\% of the 71 chips on the wafer are selected as candidates for bump processing. As the die size is large $(\sim 21 \mathrm{~mm} \times 19 \mathrm{~mm})$ this yield is considered to be acceptable. The sensor chips, which come in the form of $125 \mathrm{~mm}$ wafers from Canberra in Belgium [5] are considered to be all good. These cannot be tested pixel-by-pixel but Canberra does measure key parameters on test diodes on the wafers. The sensor wafers, CMOS wafers and wafer maps are then shipped to VTT in Finland [6]. After incoming inspection TiWCu field metal (FM) layers are deposited on the surface of the wafers. These FM layers act as an electrode for subsequent electrolytic deposition. Thick photoresist is then patterned on the wafer around the openings in the passivation where bumps are to be grown. There are $\sim 8000$ bumps on a single chip arranged as a matrix of $32 \times 256$ on pitches of $500 \mu \mathrm{m} \times 62.5 \mu \mathrm{m}$. In the standard VTT process $\mathrm{Ni}$, which is used as the under bump metallization (UBM), and eutectic solder are then electroplated on both CMOS and sensor substrates. The solder is normally grown thicker on the readout wafer side. For both the sensor and CMOS wafers, the photoresist and metallic FM layers are removed and then the wafers are heated to reflow the bumps
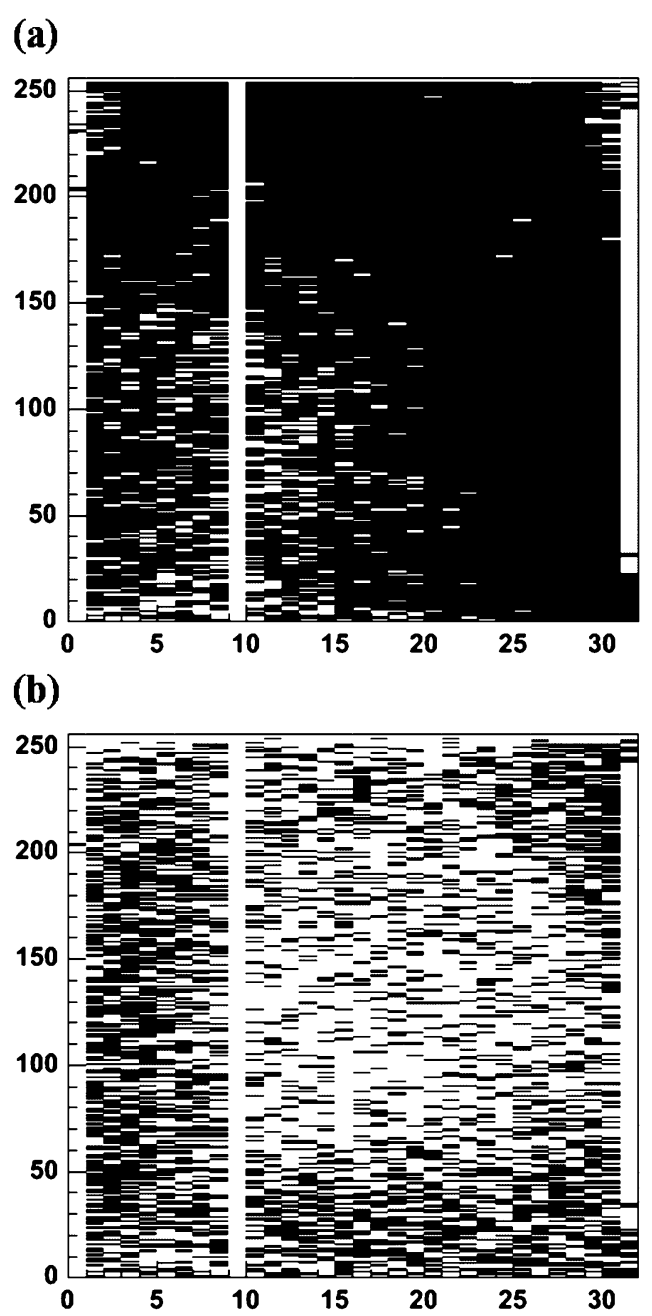

Fig. 3. Radioactive source measurements of a eutectic solder hybrid pixel detector (a) before bake out and (b) after bake out. White areas represent non-responding pixels.

and prepare them for the final bonding process. The wafers are subsequently diced and Known Good Die from the CMOS wafer are bump bonded to individual sensor chips.

The assemblies are shipped to CERN where automated pixel-by-pixel electrical tests are repeated on a probe station to ensure that there has been no degradation in the chip behaviour during bump-bonding. Furthermore, a radioactive source is used to verify the quality of the bump bonds. $\mathrm{A}^{109} \mathrm{Cd}$ source is placed on a jig above the biased assembly on the probe station illuminating the sensor with mostly $22 \mathrm{keV}$ and $25 \mathrm{keV}$ photons. These photons deposit roughly the same number of electron-hole pairs in the $\mathrm{Si}$ as a $20 \mathrm{keV}$ electron. As the ASIC has a fast-OR output this can be used to generate trigger pulses which are then sent back to the chip. Around 80000 triggers are accumulated yielding $\sim 10$ hits per pixel. The full process takes $\sim 10$ mins per assembly and limits the precision of the measurements to $\sim 99 \%$ of responding pixels, the specification for LHCb. After this the assemblies are shipped to HCM in France [7] for attachment to custom designed ceramic carriers produced by Kyocera in Germany [8] using a high curing temperature $\left(400^{\circ} \mathrm{C}\right)$ silver glass glue. The pixel photo-anodes, see Fig. 2, are then shipped back to CERN for final testing before 

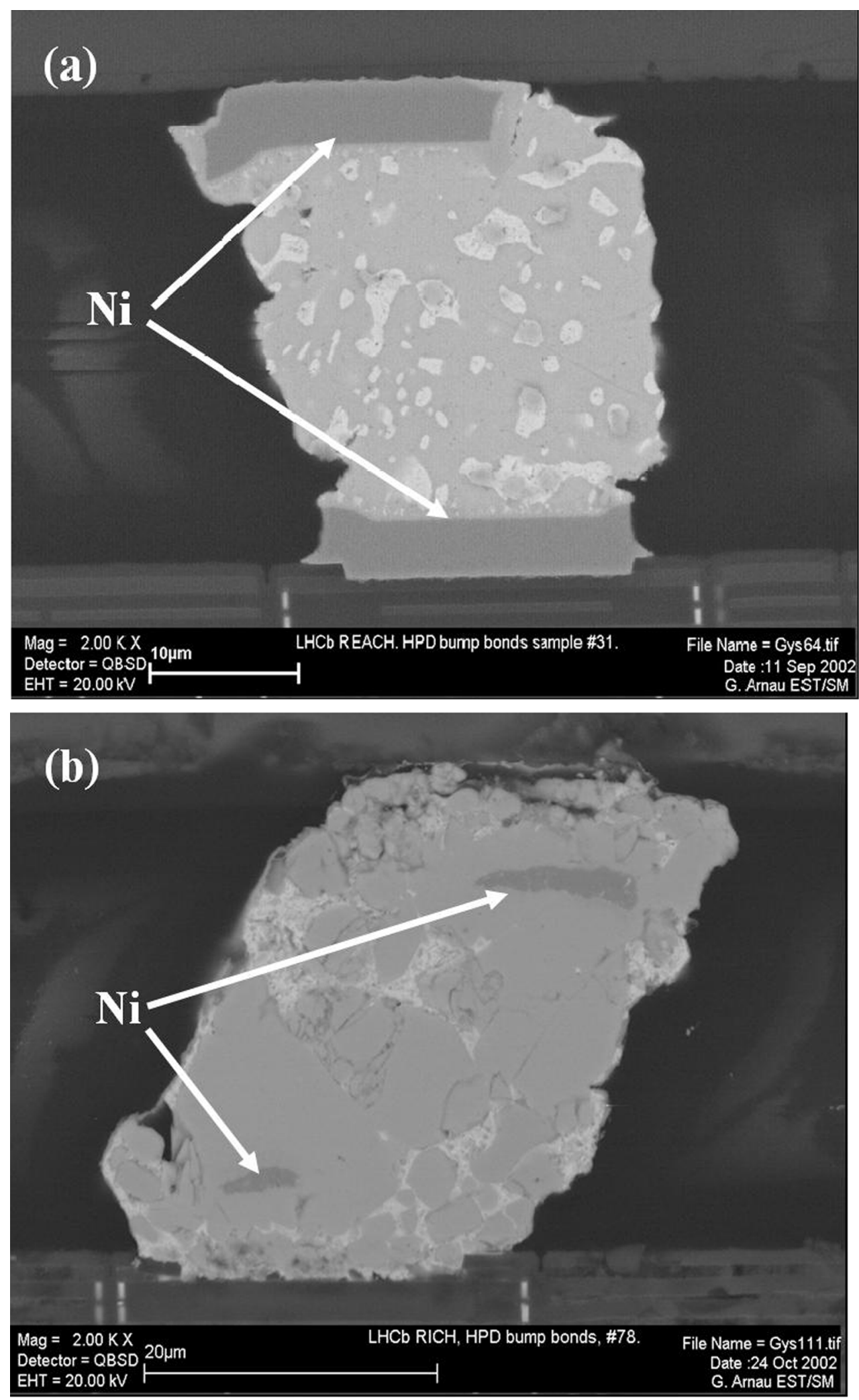

Fig. 4. (a) SEM image of the cross section of eutectic bump prior to high temperature bake out. (b) SEM image of the cross section of a similar bump post bake out.

being shipped on to DEP in The Netherlands [9] for encapsulation into the HPD tubes. The encapsulation process involves a 3 hour long bake out at $300^{\circ} \mathrm{C}$. Pixel-HPD tubes are then sent to various test centres in the LHCb RICH collaboration for final qualification prior to assembly on to the experiment's detector support. 


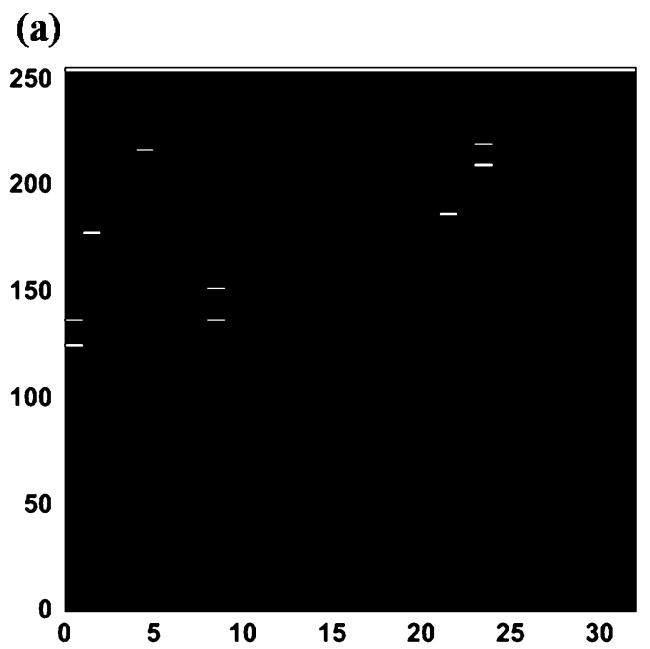

(b)

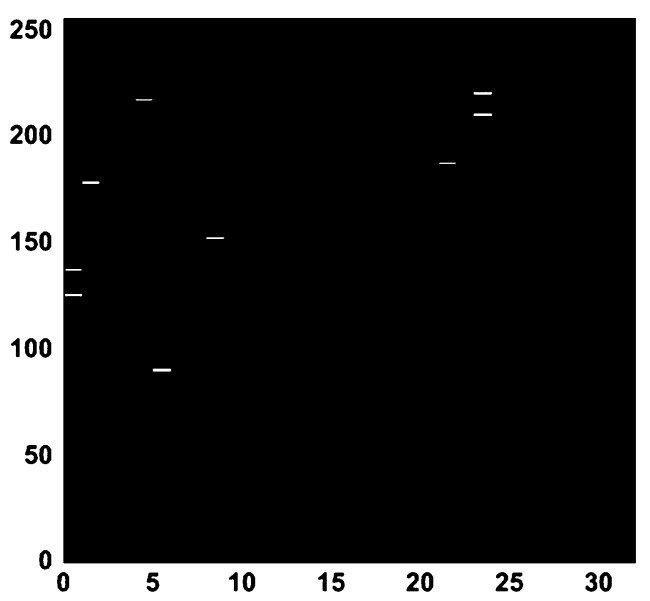

Fig. 5. Radioactive source measurements of a high-Pb solder hybrid pixel detector (a) before bake out and (b) after bake out. White areas represent non-responding pixels.

\section{MOdifiCATION TO BUMP BONDING PROCESS FOR PIXEL-HPD FABRICATION}

The melting point of eutectic solder $63 \mathrm{wt} \% \mathrm{Sn}-37 \mathrm{wt} \% \mathrm{~Pb}$ is $183^{\circ} \mathrm{C}$. As the bake out temperatures for pixel-anode fabrication and subsequent tube encapsulation are much higher than this the eutectic solder bumps would certainly melt. Fig. 3 shows the response of a eutectic solder assembly which has been mounted in a carrier to a radioactive source before and after the higher temperature bake out cycles. Note that columns 0,9 and 31 were not responding electrically during these test due to a missing wire bond (9) or incorrect electrical settings of the chip (0 and 31 ). The bake out cycles used mimic exactly the conditions expected during pixel-HPD manufacture. Although the assembly was imperfect prior to bake out there is a clear degradation in the number of responding pixels. Moreover the mechanical strength of the bonding is significantly reduced as shown elsewhere [10]. In order to understand better the intermetallic processes taking place two assemblies were cut vertically through the bumps and SEM images produced. The results are shown in Fig 4. Prior to bake out the Ni UBM "plugs" are well defined and separated from the $\mathrm{Sn}-\mathrm{Pb}$ eutectic mixture. After bake out, however, the Ni UBM has delaminated and almost completely dissolved in the Sn rich solder mixture. From these photos it was evident that eutectic solder was not a viable solution for the pixel-HPD assembly procedure.

VTT proposed to make changes to their standard bump bonding process in order to meet this new challenge. Instead of depositing only thick eutectic solder on the readout wafer they proposed to deposit a thin eutectic layer followed by a thick pure $\mathrm{Pb}$ layer. This required the preparation of a new $\mathrm{Pb}$ only electrolytic bath and a number of extra processing steps to avoid oxidation of the high- $\mathrm{Pb}$ solder bump. The eutectic composition of the bump on the detector side remains unchanged. The aim was to obtain a final bump composition after flip-chip assembly of $10 \mathrm{wt} \% \mathrm{Sn}-90 \mathrm{wt} \% \mathrm{~Pb}$ melting at $\sim 300^{\circ} \mathrm{C}$

\section{Results of Assembly Tests Using High-Pb Content BUMPS}

A radioactive source measurement from one of the first assemblies produced using the high- $\mathrm{Pb}$ solder is shown in Fig. 5(a). It was also subjected to the standard bake out cycle and, as shown in Fig. 5(b), the bumps withstand the stress rather well. As proof of this two high- $\mathrm{Pb}$ assemblies were cut through the bumps and SEM photos taken. The results are shown in 

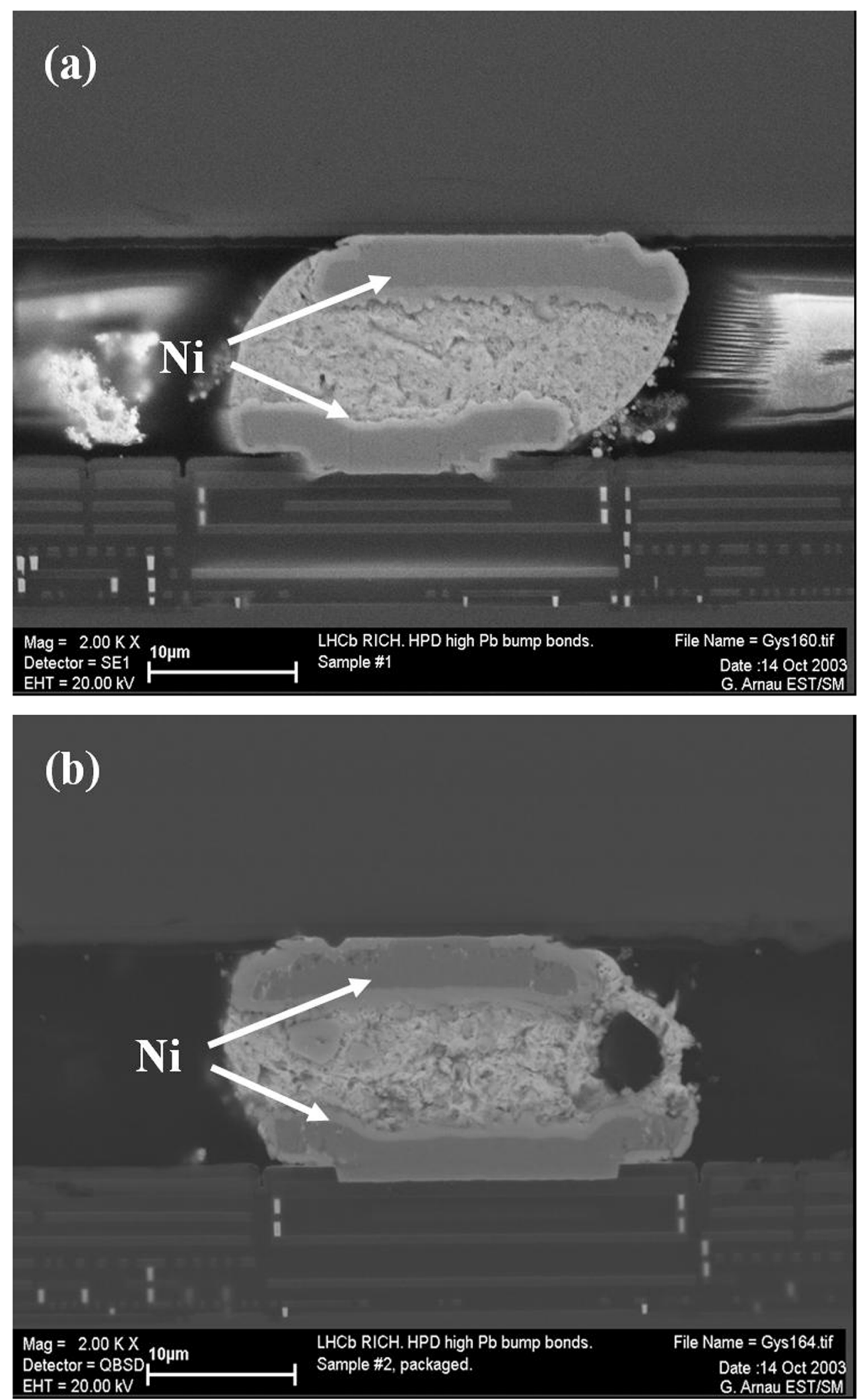

Fig. 6. (a) SEM image of the cross section of high-Pb bump prior to high temperature bake out. (b) SEM image of the cross section of a similar bump post bake out.

Fig 6. It is clear from Fig 6(b) that the high-Pb solder does not dissolve the Ni UBM and the bumps remain in place.

More than 40 hybrid pixel assemblies have been produced using the new high- $\mathrm{Pb}$ bump-bonding process. Of those over
$90 \%$ had less than $1 \%$ of bumps missing after fabrication. The other $10 \%$ of assemblies were rejected mainly because of electrical chip failures, probably due to problems with electrostatic discharge. Six good assemblies were baked out and 


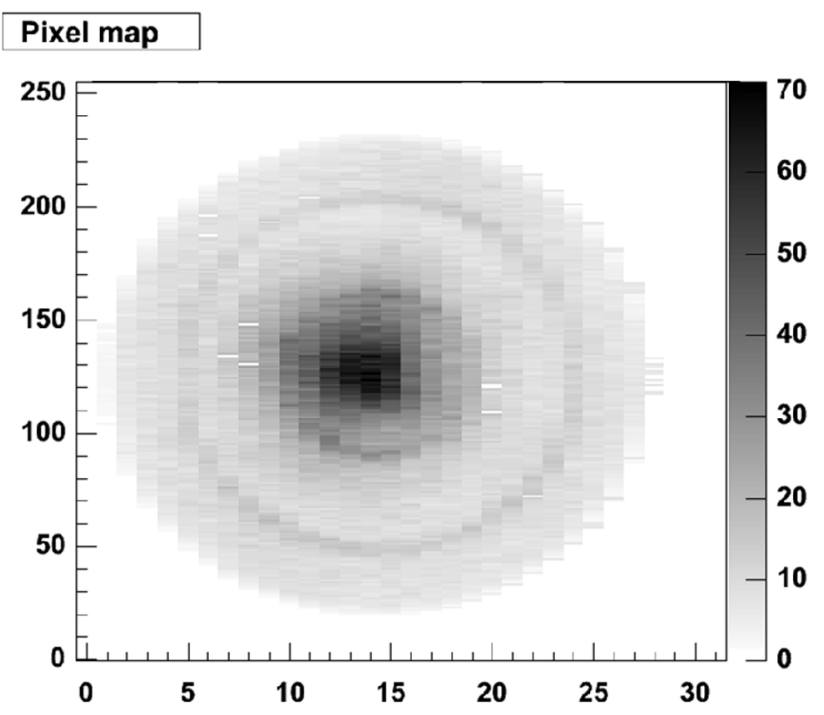

Fig. 7. The response of a pixel-HPD stimulated with an LED. A few missing bumps are evident in the centre of the tube, but otherwise the response is almost perfectly uniform.

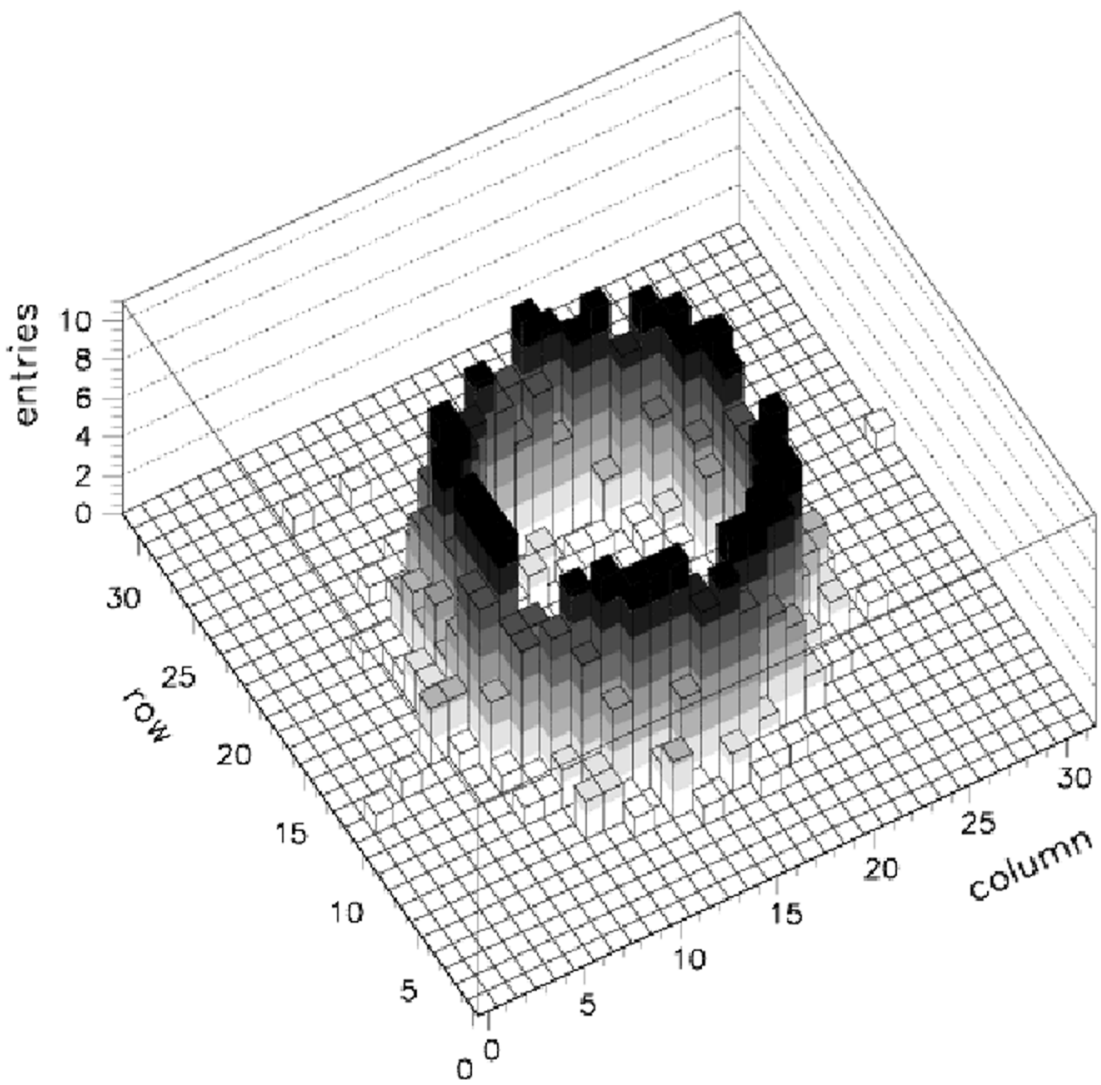

Fig. 8. Cherenkov rings from (mainly) $10 \mathrm{GeV}$ pions traversing an air volume focused on a pixel-HPD tube [11].

none of these demonstrated any degradation as a consequence. Finally 10 prototype pixel-HPD tubes have been manufactured and Fig. 7 indicates how one of these reacts to stimulus with an LED. The performance of the pixel-HPD's was verified in a test beam revealing very clean Cherenkov rings [11], see Fig. 8.

\section{CONCLUSION}

A new high-Pb fine pitch bump bonding process has been developed. Tests with individual assemblies and measurements on a sizable number of prototypes suggest that the bump bonding process is not only well-mastered but fulfills the requirements for the production of the pixel-HPD's for the LHCb RICH detector. Production of the full series of 800 assemblies is now underway.

\section{REFERENCES}

[1] LHCb Technical Proposal. The LHCb Collaboration, Feb. 20, 1998, CERN/LHCC 98-4, LHCC/P4. 
[2] LHCb RICH Technical Design Report. The LHCb Collaboration, Sep. 7, 2000, CERN/LHCC 2000-037, LHCb TDR 3.

[3] T. Gys, "The pixel hybrid photon detectors for the LHCb-RICH project," Nucl. Instrum. Methods Phys. Res. A, vol. A465, no. 1, pp. 240-6, Jun. 1, 2001.

[4] K. Wyllie, G. Anelli, F. Bal, M. Burns, M. Campbell, M. C. Carballo, P. Chochula, R. Dinapoli, F. Formenti, M. Girone, A. G. Elias, T. Gys, S. Jolly, A. Kluge, M. Morel, H. Muller, P. Riedler, D. S. S. Bello, W. Snoeys, G. Stefanini, J. van Hunen, and E. Watson, "A pixel readout chip for tracking at ALICE and particle identification at LHCb," in Proc. 5th Workshop Electronics for LHC Experiments, Snowmass, CO, Sep. 20-24, 1999, pp. 93-97, CERN/LHCC 99-33.

[5] “Canberra Semiconductors N.V.," Belgium, Lammerdries 25, B-2250 Olen.

[6] “VTT Information Technology," Finland, Teitotie 3, FIN-02044 VTT Espoo.
[7] “Hybritech Composants Microelectronique," France, Z. I. De Perigny, Av. Joliot-Curie, F-17185 Perigny CEDEX.

[8] “Kyocera Fineceramics GmbH,” Germany, Fritz-Mueller Strasse 107, D-73730 Esslingen.

[9] "Delft Electronic Products B.V.," The Netherlands, Dwazziewegen 2, NL-9300 AB Roden.

[10] M.-L. Delsante, G. A. Izquierdo, and T. Gys, "Investigation of Sn-Pb solder bumps of prototype photo detectors for the LHCb experiment," J. Mater. Process. Technol., vol. 155-156, pp. 1668-1672, Nov. 30, 2004.

[11] M. Moritz, G. Aglieri Rinella, L. Allebone, M. Campbell, T. Gys, C. Newby, A. Pickford, D. Piedigrossi, and K. Wyllie, "Performance study of new pixel hybrid photon detector prototypes for the LHCb RICH counters," IEEE Trans. Nucl. Sci., vol. 51, no. 3, p. 1060, Jun. 2004. 